\title{
Estimating effective boundaries of population growth in a variable environment
}

\author{
Vladimir Kozlov', Sonja Radosavljevic ${ }^{1 *} \mathbb{D}$, Bengt Ove Turesson ${ }^{1}$ and Uno Wennergren²
}

"Correspondence:

sonja.radosavljevic@liu.se

'Department of Mathematics, Linkoping University, Linkoping, 58381, Sweden

Full list of author information is available at the end of the article

\begin{abstract}
We study the impact of age-structure and temporal environmental variability on the persistence of populations. We use a linear age-structured model with time-dependent vital rates. It is the same as the one presented by Chipot in (Arch. Ration. Mech. Anal. 82(1):13-25, 1983), but the assumptions on the vital rates are slightly different. Our main interest is in describing the large-time behavior of a population provided that we know its initial distribution and transient vital rates. Using upper and lower solutions for the characteristic equation, we define time-dependent upper and lower boundaries for a solution in a constant environment. Moreover, we estimate solutions for the general time-dependent case and also for a special case when the environment is changing periodically.
\end{abstract}

MSC: 35B40; 35C15; 92D25

Keywords: age-structure; time-dependency; environmental variability; population growth; upper and lower bounds; periodic oscillations

\section{Introduction}

We live in the age when the number of rare or nearly extinct species is growing daily. Climate change and environmental pollution caused by human activity have profound impact on the extinction risk for many species; see, e.g., [2]. In order to avert the possible extinction and preserve the diversity in nature, we need to understand how temporal environmental changes influence the birth and death rates of individuals in a population, and by influencing the birth and death rates, how these changes influence population growth.

When it comes to the permanence of a population, safety is not always in numbers because environmental variation affects a population regardless of its size. Moreover, one of the main concerns for conservation biologists is in endangered species. This means that small populations are encountered in most cases, and for them demography plays a much more prominent role in comparison to large populations. In other words, the risk of extinction due to demography for small populations is high.

The conclusion that arises is that both demography and temporal environmental variability need to be considered when we set up a population model. Demography is traditionally introduced in population models through age-structure. One of the first such models was developed by Sharpe and Lotka [3] in 1911, McKendrick [4] in 1926, and von Foerster [5] in 1959. This model describes population dynamics as a linear process. A central place has the age-class density function $n(a, t)$, where $a$ is the age of an individual and

(c) 2016 Kozlov et al. This article is distributed under the terms of the Creative Commons Attribution 4.0 International License (http://creativecommons.org/licenses/by/4.0/), which permits unrestricted use, distribution, and reproduction in any medium, provided you give appropriate credit to the original author(s) and the source, provide a link to the Creative Commons license, and indicate if changes were made. 
$t$ is the time. A population grows (or declines) exponentially, which is defined by agedependent birth and death rates through the Lotka-Euler characteristic equation. A solution to the characteristic equation is related to the net reproductive rate and it uniquely determines population dynamics. For more details and analysis of the continuous linear model we refer the reader to [6-9], and [10]. There is also interest for studying nonlinear age-dependent models, in which case the vital rates depend on the age-class and on the population density; see for instance $[9,11]$ and [12]. An analysis of discrete population models can be found in [13].

From the ecological point of view, it is crucially important to study the interplay between age-structure and environmental variation and describe how combination of these two factors influence population growth. Besides, based on the information as regards transient vital rates, a model should be able to predict population dynamics for any time. This is translated into an analysis of the large-time behavior of a solution to an age-structured time-dependent model.

The linear time-independent model, mentioned above, is easy to use and on many occasions it gives satisfactory results. Unfortunately, the model lacks the ability to deal with environmental fluctuations and its effects on population growth. To encompass environmental variability, time-dependency must be included to a model. We refer to Chipot in [1] where the linear theory developed by Sharpe and Lotka and McKendrick is extended through time-dependent vital rates. Under certain assumptions on the vital rates, using a fixed point argument, he proved that the model has a unique nonnegative solution. Cushing in [14] studied existence of time-periodic solutions to the model under specific assumptions on the vital rates.

In order to investigate how transient time-dependent vital rates affect dynamics of an age-structured population, we use the same model as Chipot in [1]. Under slightly different conditions for the vital rates we prove the existence and uniqueness of a solution. However, the main part of our work is dedicated to the analysis of its large-time behavior.

Since the vital rates are now time dependent, the characteristic equation is difficult, if not impossible, to solve analytically. Ecologically, not knowing the exact solution of the problem can be compensated for by considering the boundaries within which a solution fluctuates in time. Very low boundaries indicate the risk of extinction and very high boundaries can imply unrestrained increase.

Using the method of upper and lower solutions for integral equations makes solving the characteristic equation redundant. We obtain upper and lower solutions for the characteristic equation instead and use them to determine upper and lower bounds for the number of newborns and the total population. These upper and lower bounds are time-dependent functions that correspond to the best case scenario and to the worst case scenario, respectively. Provided that the initial distribution of a population and the vital rates are known, we estimate the population density in the worst and in the best case scenarios. Naturally, in all other cases the population density lies between boundaries given by the population densities in these two extreme cases. As in the time-independent case, the population exhibits exponential growth or decline, depending on the vital rates.

Temporal environmental variability quite often presuppose periodic changes. Under the assumption that the birth rate is a time-periodic function and the death rate is a timeindependent function, we obtain an exact upper and lower bound for the number of newborns and the total population. Tuljapurkar in [15] presents a similar study for a discrete 
linear population model and proves that population growth is governed by the average vital rates. He claims that the growth rate is increased by oscillations with periods near the generation time and decreased by oscillations with much shorter or much longer periods.

Our analysis also concludes that the average vital rates determine population growth. However, if a population has zero intrinsic growth rate, the frequency of oscillation can cause growth or decline, depending on the life history. Oscillations with very low frequencies are detrimental for population growth for all observed life histories. Unlike Tuljapurkar, we show that if the period is comparable to the generation time, one needs to consider life history as well since different species having different responses to changes in the environment. This observation implies that there is a deeper connection between age-structure and time dependency that should be investigated.

\section{Age-structured model in a variable environment}

The model we are going to use is basically the same as in [1]. We keep the same equations, but some of the assumptions are changed. The balance equation for this model is

$$
\frac{\partial n(a, t)}{\partial t}+\frac{\partial n(a, t)}{\partial a}=-\mu(a, t) n(a, t), \quad a, t>0,
$$

and the boundary and initial conditions are given by

$$
\begin{aligned}
& n(0, t)=\int_{0}^{\infty} m(a, t) n(a, t) d a, \quad t>0, \\
& n(a, 0)=f(a), \quad a>0,
\end{aligned}
$$

where $n(a, t)$ is the number of individuals in the age class $a$ at time $t, m(a, t)$, and $\mu(a, t)$ are their birth and death rate, respectively, and $f(a)$ is the initial distribution of the population into age classes. However, in order to have a biologically meaningful model, the functions that appear must meet certain requirements. We have the following assumptions: (1) the constant $A_{\mu}$ denotes an upper bound for the maximal length of life of individuals in population, the constant $A_{m}$ is the upper bound of fertility period and $A_{m} \leq A_{\mu}$, (2) the birth rate $m$, the death rate $\mu$, and the initial distribution $f$ of a population into age classes are measurable and nonnegative functions with the following properties:

(i) $\quad m(a, t)$ is bounded for $a, t \geq 0$, $m(a, t)=0$ for $a>A_{m}$ and $t \geq 0$, $m(a, t) \geq \delta_{1}>0$ for $a_{1}<a<a_{2}$, where $0<a_{1}<a_{2}<A_{m}$ and $t \geq 0$,

(ii) $\quad 0<c_{\mu} \leq \mu(a, t) \leq C_{\mu}<\infty$ for $a \leq a_{2}$ and $t \geq 0$, $\int_{A}^{A+A_{\mu}} \mu(a, t) d a=\infty$ for $t \geq 0$ and $A \geq 0$,

(iii) $f$ is bounded,

$$
\begin{aligned}
& f(a) \geq \delta_{2}>0 \text { for } b_{1}<a<b_{2}, \text { where } 0<b_{1}<b_{2}<a_{2}, \\
& f(a)=0 \text { for } a>A_{\mu} .
\end{aligned}
$$

Our aim is to prove that the model with this assumption has a unique nonnegative solution. ${ }^{a}$ We begin by showing that if a solution $n$ to the problem (1)-(3) exists, it satisfies a certain integral equation.

Theorem 2.1 Let $n \in C^{1}((0, \infty) \times(0, \infty))$, be a solution to the population model (1)-(3). Then the function

$$
\rho(t)=n(0, t), \quad t>0,
$$


satisfies the integral equation

$$
\begin{aligned}
\rho(t)= & \int_{0}^{t} m(a, t) e^{-\int_{0}^{a} \mu(v, v+t-a) d v} \rho(t-a) d a \\
& +\int_{t}^{\infty} m(a, t) e^{-\int_{a-t}^{a} \mu(v, v+t-a) d v} f(a-t) d a
\end{aligned}
$$

for $t>0$. For $a>0, n(a, t)$ is given by

$$
n(a, t)= \begin{cases}\rho(t-a) e^{-\int_{0}^{a} \mu(v, v+t-a) d v}, & 0<a<t, \\ f(a-t) e^{-\int_{a-t}^{a} \mu(v, v+t-a) d v}, & a \geq t .\end{cases}
$$

This theorem allows us to introduce a generalized solution to the problem (1)-(3), which is given by (5), where $\rho \in L_{\mathrm{loc}}^{\infty}[0, \infty)$ satisfies (4). Its proof is quite standard, but for completeness we give it in the Appendix.

In order to show that the model (1)-(3) has a unique solution, it is sufficient to prove that equation (4) has a unique solution. For this purpose we use the Banach fixed point theorem. Namely, if $\rho$ is a solution to equation (4), it can be written in the following way:

$$
\rho(t)=K \rho(t)+F(t), \quad t \geq 0,
$$

where

$$
K \rho(t)=\int_{0}^{t} m(a, t) e^{-\int_{0}^{a} \mu(v, v+t-a) d v} \rho(t-a) d a, \quad t \geq 0,
$$

and

$$
F(t)=\int_{t}^{\infty} m(a, t) e^{-\int_{a-t}^{a} \mu(v, v+a-t) d v} f(a-t) d a, \quad t \geq 0 .
$$

Given a positive real number $\Lambda$, let $L_{\Lambda}^{\infty}(0, \infty)$ denote the space of measurable functions $u$ on $[0, \infty)$ such that $|u(t)|=O\left(e^{\Lambda t}\right)$ for $t \geq 0$. The norm on $L_{\Lambda}^{\infty}(0, \infty)$ is defined by

$$
\|u\|_{\Lambda}=\underset{t>0}{\operatorname{ess} \sup }|u(t)| e^{-\Lambda t}
$$

and $L_{\Lambda}^{\infty}(0, \infty)$ is Banach space for every positive real $\Lambda$.

We first show that the operator $K$ is a contraction on $L_{\Lambda}^{\infty}(0, \infty)$ for sufficiently large $\Lambda$. For brevity, we use the notation

$$
Q(a, t)=m(a, t) e^{-\int_{0}^{a} \mu(v, v+t-a) d v}, \quad a, t \geq 0 .
$$

The assumptions (i)-(ii) imply that

$$
Q(a, t) \geq \delta_{1} e^{-C_{\mu} a_{2}}>0 \quad \text { for } a \in\left(a_{1}, a_{2}\right) \text { and } t \geq 0 .
$$

In the next lemma we gather some properties of the function $F$.

Lemma 2.2 For a function $F$ defined by (8), the following properties hold: 
(1) There exist an interval $\left(t_{1}, t_{2}\right) \subset\left(0, a_{2}-b_{1}\right)$ and a positive number $\delta$ such that

$$
F(t) \geq \delta \quad \text { for } t \in\left(t_{1}, t_{2}\right)
$$

(2) $F(t)=0$ for $t \geq A_{m}$;

(3) $F$ is a bounded function on $[0, \infty)$.

Proof (1) According to the assumptions (i)-(iii) and (9), we have

$$
\begin{aligned}
F(t) & \geq \delta_{1} e^{-C_{\mu} a_{2}} \int_{a_{1}}^{a_{2}} f(a-t) d a=\delta_{1} e^{-C_{\mu} a_{2}} \int_{a_{1}-t}^{a_{2}-t} f(x) d x \\
& \geq \delta_{1} \delta_{2} e^{-C_{\mu} a_{2}} \int_{\max \left(a_{1}-t, b_{1}\right)}^{\min \left(a_{2}-t, b_{2}\right)} d x \\
& \geq \delta_{1} \delta_{2} e^{-C_{\mu} a_{2}} \min \left(a_{2}-a_{1}, a_{2}-b_{1}-t_{2}, b_{2}-a_{1}+t_{1}, b_{2}-b_{1}\right)>0
\end{aligned}
$$

for $t \in\left(t_{1}, t_{2}\right) \subset\left(0, a_{2}-b_{1}\right)$.

(2) Since $m(a, t)=0$ for $a$ outside of $\left(0, A_{m}\right)$, it follows that $F(t)=0$ for $t \geq A_{m}$.

(3) By (i)-(iii), the functions $m$ and $f$ are bounded. Let $m^{*}=\sup _{a, t \geq 0} m(a, t)$ and $f^{*}=$ $\sup _{a \geq 0} f(a)$. Then we have

$$
|F(t)| \leq e^{-c_{\mu} t} \int_{t}^{\infty} m(a, t) f(a-t) d a \leq e^{-c_{\mu} t} f^{*} \int_{0}^{A_{m}} m(a, t) d a \leq e^{-c_{\mu} t} f^{*} m^{*} A
$$

which proves the claim.

Lemma 2.3 The operator $K$ is a contraction on $L_{\Lambda}^{\infty}(0, \infty)$ provided that $\Lambda$ is sufficiently large.

Proof Let $\rho \in L_{\Lambda}^{\infty}(0, \infty)$. Since the function $m$ is bounded and $\mu$ is nonnegative, there exists a constant $Q^{*} \geq 0$ such that

$$
Q(a, t) \leq Q^{*} \text { for } a, t \geq 0
$$

We therefore have

$$
\begin{aligned}
|K \rho(t)| e^{-\Lambda t} & \leq \int_{0}^{t} Q(a, t)|\rho(t-a)| e^{-\Lambda t} d a \\
& \leq \int_{0}^{t} Q^{*}|\rho(t-a)| e^{-\Lambda(t-a)} e^{-\Lambda a} d a \\
& \leq Q^{*} \frac{1-e^{-\Lambda t}}{\Lambda}\|\rho\|_{\Lambda}
\end{aligned}
$$

for $t \geq 0$, and, consequently,

$$
\|K \rho\|_{\Lambda} \leq \frac{Q^{*}}{\Lambda}\|\rho\|_{\Lambda}
$$

This shows that $K \rho \in L_{\Lambda}^{\infty}(0, \infty)$. Moreover, if $\Lambda>Q^{*}$, then the operator $K$ is a contraction. 
Using this lemma, we prove the main result of this section.

Theorem 2.4 Equation (6) has a unique solution $\rho \in L_{\Lambda}^{\infty}(0, \infty)$ provided that $\Lambda$ is suffciently large.

Proof To prove the claim, it is sufficient to notice that the operator $K$ is a contraction on $L_{\Lambda}^{\infty}(0, \infty)$ for large values of $\Lambda$ due to Lemma 2.3 and that $F \in L^{\infty}(0, \infty)$ by Lemma 2.2. The result follows from the Banach fixed point theorem.

Remark 2.5 If we additionally assume that $F$ and $Q$ are continuous functions with respect to $t$, then the solution $\rho$ to (6) is continuous with respect to $t$.

Remark 2.6 A solution to the fixed point equation (6) is the limit of the monotonically increasing sequence

$$
\rho_{k+1}=K \rho_{k}+F, \quad \rho_{0}=0, \quad k \geq 1 .
$$

Due to the assumptions (i)-(iii), every term in the sequence is nonnegative, which implies that the solution is nonnegative.

To see how small perturbations in the birth rate affect the stability of the model, we consider the function $m$ as a parameter. From equation (6), we obtain the following integral equation:

$$
\rho_{m}(t)=K_{m} \rho_{m}(t)+F_{m}(t), \quad t \geq 0
$$

where the index $m$ means that the function $m$ is a parameter.

Let $P$ be a set of functions $m$ that satisfy (i) and that are uniformly bounded on $\mathbb{R}^{+} \times \mathbb{R}^{+}$ by some constant $C>0$. Then $P$ is a metric space with the metric borrowed from $L^{\infty}\left(\mathbb{R}^{+} \times\right.$ $\left.\mathbb{R}^{+}\right)$. We denote by $\rho_{m}, m \in P$, the unique solution in $L_{\Lambda}^{\infty}(0, \infty)$ to equation (11).

Theorem 2.7 If $m \rightarrow m_{0}$ in $P$, then $\rho_{m} \rightarrow \rho_{m_{0}}$ in $L_{\Lambda}^{\infty}(0, \infty)$ provided that $\Lambda$ is sufficiently large.

Details and proof of this theorem can be found in the Appendix.

A similar conclusion is valid under the assumption that the initial distribution of population, $f(a)$, undergo small perturbations.

\section{General upper and lower bounds}

The main purpose of our investigations of the model (1)-(3), or equations (4) and (5), is to explain the interplay between time dependence in the vital rates and population growth. Earlier models use time-independent vital rates and make predictions of population growth or decline by solving and analyzing the so-called Lotka-Euler characteristic equation,

$$
\int_{0}^{\infty} Q(a) e^{-k a} d a=1
$$


The parameter $k$ obtained from this equation is the intrinsic growth rate and the following holds: $N(t) \rightarrow \infty$ for $k>0$, while $N(t) \rightarrow 0$ for $k<0$.

Due to the fact that the birth and death rate are now functions of age and time, we expect a different and more complex characteristic equation. Assuming that the solution to equation (7) is of the form $e^{\int_{0}^{t} \sigma(\tau) d \tau}$ and $M>A_{m}$, we get the characteristic equation

$$
\int_{0}^{\infty} Q(a, t) e^{-\int_{t-a}^{t} \sigma(\tau) d \tau} d a=1 \quad \text { for } t \geq M
$$

In analogy to the analysis that followed from the Lotka-Euler characteristic equation, we base our predictions of population growth on equation (13) and the function $\sigma$. Since solving equation (13) can be a difficult task, we agree to the following trade-off: instead of solving the original problem (i.e., finding a fixed point of equation (6)), we are looking for upper and lower bounds of equation (6).

Definition 3.1 A nonnegative function $\rho^{+} \in L_{\Lambda}^{\infty}(0, \infty)$ is an upper solution to equation (6) if

$$
\rho^{+}(t) \geq K \rho^{+}(t)+F(t) \quad \text { for } t \geq 0 .
$$

Similarly, a nonnegative function $\rho^{-} \in L_{\Lambda}^{\infty}(0, \infty)$ is a lower solution to equation (6) if

$$
\rho^{-}(t) \leq K \rho^{-}(t)+F(t) \quad \text { for } t \geq 0
$$

The importance of this definition is that the upper and lower solutions give upper and lower bounds for the function $\rho(t)$ for $t \geq 0$. Namely, the following holds:

$$
\rho^{-}(t) \leq \rho(t) \leq \rho^{+}(t) \quad \text { for } t \geq 0
$$

For the details, see Section 7.4 in [16].

The next result is about upper solutions to equation (6).

Theorem 3.2 Suppose that $M \geq A_{m}$ and let $\rho$ be a solution to equation (6). If the function $\sigma \in L^{\infty}(0, \infty)$ satisfies

$$
\int_{0}^{\infty} Q(a, t) e^{-\int_{t-a}^{t} \sigma(\tau) d \tau} d a \leq 1 \quad \text { for } t \geq M
$$

then there exists a constant $D>0$ such that

$$
\rho(t) \leq D e^{\int_{0}^{t} \sigma(\tau) d \tau} \quad \text { for } t \geq M .
$$

Proof Let

$$
f_{1}(a)= \begin{cases}e^{-\sigma_{1} a}, & 0 \leq a \leq M, \\ 0, & a>M,\end{cases}
$$


and

$$
\rho^{+}(t)= \begin{cases}D_{1} e^{\sigma_{1} t}, & 0 \leq t \leq M \\ D_{2} e^{\int_{0}^{t} \sigma(\tau) d \tau}, & t>M\end{cases}
$$

where the constants $\sigma_{1}, D_{1}$, and $D_{2}$ will be determined later. To prove the claim, we first show that the function $\rho^{+}$is an upper solution to equation (6) with $f=f_{1}$.

For $0 \leq t \leq M$, we have

$$
\begin{aligned}
\rho^{+}(t)-K \rho^{+}(t)-F_{1}(t)= & D_{1} e^{\sigma_{1} t}-D_{1} \int_{0}^{t} Q(a, t) e^{\sigma_{1}(t-a)} d a \\
& -\int_{t}^{\infty} m(a, t) e^{-\int_{a-t}^{a} \mu d v} e^{-\sigma_{1}(a-t)} d a \\
= & D_{1} e^{\sigma_{1} t}\left(1-\int_{0}^{t} Q(a, t) e^{-\sigma_{1} a} d a\right) \\
& -e^{\sigma_{1} t} \int_{t}^{\infty} m(a, t) e^{-\int_{a-t}^{a} \mu d v} e^{-\sigma_{1} a} d a,
\end{aligned}
$$

where $F_{1}$ is evaluated by the right-hand side of (8) with $f$ replaced by $f_{1}$. Due to the assumptions (i)-(ii), there exist constants $c_{1}$ and $c_{2}$ such that $Q(a, t) \leq c_{1}$ and $m(a, t) \leq c_{2}$ for all $a, t \geq 0$. This implies that

$$
\int_{0}^{M} Q(a, M) e^{-\sigma_{1} a} d a \leq \frac{c_{1}}{\sigma_{1}} \text { and } \int_{0}^{\infty} m(a, 0) e^{-\sigma_{1} a} d a \leq \frac{c_{2}}{\sigma_{1}}
$$

Thus, if the constants $D_{1}$ and $\sigma_{1}$ satisfy

$$
D_{1} \geq D_{1} \frac{c_{1}}{\sigma_{1}}+\frac{c_{2}}{\sigma_{1}}
$$

then we have $\rho^{+}-K \rho^{+}-F_{1} \geq 0$ on $[0, M]$.

Let $t>M$. According to Lemma 2.2, $F_{1}(t)=0$ for $t \geq A_{m}$, which implies that

$$
\begin{aligned}
\rho^{+}(t)-K \rho^{+}(t)= & D_{2} e^{\int_{0}^{t} \sigma(\tau) d \tau}-D_{1} \int_{t-M}^{t} Q(a, t) e^{\sigma_{1}(t-a)} d a \\
& -D_{2} \int_{0}^{t-M} Q(a, t) e^{\int_{0}^{t-a} \sigma(\tau) d \tau} d a \\
= & D_{2}\left(e^{\int_{0}^{t} \sigma(\tau) d \tau}-\int_{0}^{\infty} Q(a, t) e^{\int_{0}^{t-a} \sigma(\tau) d \tau} d a\right) \\
& +D_{2} \int_{t-M}^{\infty} Q(a, t) e^{\int_{0}^{t-a} \sigma(\tau) d \tau} d a-D_{1} \int_{t-M}^{t} Q(a, t) e^{\sigma_{1}(t-a)} d a .
\end{aligned}
$$

The assumption (14) guarantees that the difference in (15) is nonnegative. Equation (16) is nonnegative if

$$
D_{2} e^{\int_{0}^{t} \sigma(\tau) d \tau} \geq D_{1} e^{\sigma_{1} t} \quad \text { for } 0 \leq t \leq M
$$

This proves that $\rho^{+}$is an upper solution to equation (6) with $f=f_{1}$ for a suitable choice of the constants $D_{1}, D_{2}$, and $\sigma_{1}$. 
Let $\rho_{1}$ be a solution to equation (6) for $f=f_{1}$. According to the properties of the upper solutions, $\rho_{1} \leq \rho^{+}$holds. By Theorem 2.4, the solutions $\rho$ and $\rho_{1}$ are obtained by the iterations (10). Since $f \leq C f_{1}$ on $\left[0, A_{\mu}\right]$ for some constant $C>0$ and both of them are zero outside of this interval, by Lemma 2.2 we have $C F_{1}-F \geq 0$. Positivity of $K$ implies $K\left(C F_{1}-F\right)(t) \geq 0$. Continuing the iterations we get

$$
C \rho_{1}(t)-\rho(t)=\sum_{j=0}^{\infty} K^{j}\left(C F_{1}-F\right)(t) \geq 0 \quad \text { for } t \geq 0
$$

and consequently

$$
\rho(t) \leq C \rho_{1}(t) \leq C \rho^{+}(t) \quad \text { for } t \geq 0
$$

which completes the proof.

The following theorem deals with the problem of finding a lower solution to equation (6). In combination with the previous result, it allows us to describe boundaries for the density of newborns for large time $t$.

Theorem 3.3 Suppose that $M \geq A_{m}$ and let $\rho$ be a solution to equation (6). If the function $\sigma \in L^{\infty}(0, \infty)$ satisfies

$$
\int_{0}^{\infty} Q(a, t) e^{-\int_{t-a}^{t} \sigma(\tau) d \tau} d a \geq 1 \quad \text { for } t \geq M
$$

then there exists a constant $C>0$ such that

$$
\rho(t) \geq C e^{\int_{0}^{t} \sigma(\tau) d \tau} \quad \text { for large } t
$$

Proof Let $H(t)$ be the Heaviside function and suppose that $\rho_{j}(t)$ is obtained by iterations

$$
\rho_{j+1}(t)=K \rho_{j}(t)+F(t), \quad \rho_{0}(t)=F(t) \quad \text { for } t \geq 0 .
$$

To prove the claim, it is sufficient to show that the function $\rho^{-}(t)$, defined by

$$
\rho^{-}(t)=\rho_{j}(t)+C e^{\int_{0}^{t} \sigma(\tau) d \tau} H(t-M), \quad t \geq 0,
$$

is a lower solution to equation (6) provided that $j$ is large enough and $C$ is a small positive constant. To this end, we estimate the expression $\rho^{-}(t)-K \rho^{-}(t)-F(t)$ from above for $t \geq 0$.

For $t<M$, the second term in the right-hand side of (19) is zero and hence

$$
\rho^{-}(t)-K \rho^{-}(t)-F(t)=\rho_{j}(t)-K \rho_{j}(t)-F(t)=-K^{j+1} F \leq 0 .
$$

For $t \geq M$, the following is true:

$$
\begin{aligned}
& \rho^{-}(t)-K \rho^{-}(t)-F(t) \\
& \quad=C e^{\int_{0}^{t} \sigma(\tau) d \tau}-C \int_{0}^{t} Q(a, t) e^{\int_{0}^{t-a} \sigma(\tau) d \tau} H(t-a-M) d a-K^{j+1} F(t) .
\end{aligned}
$$


Since $Q(a, t)=0$ for $a>A_{m}$ and $H(t-a-M)=1$ for $a \leq t-M$, it follows that

$$
\int_{0}^{t} Q(a, t) e^{\int_{0}^{t-a} \sigma(\tau) d \tau} H(t-a-M) d a=\int_{0}^{t-M} Q(a, t) e^{e_{0}^{t-a} \sigma(\tau) d \tau} d a
$$

and

$$
\begin{aligned}
\rho^{-}(t)-K \rho^{-}(t)-F(t)= & C e^{\int_{0}^{t} \sigma(\tau) d \tau}-C \int_{0}^{\infty} Q(a, t) e^{\int_{0}^{t-a} \sigma(\tau) d \tau} d a \\
& +C \int_{t-M}^{A_{m}} Q(a, t) e^{\int_{0}^{t-a} \sigma(\tau) d \tau} d a-K^{j+1} F(t) .
\end{aligned}
$$

According to the condition (17), we have

$$
\begin{aligned}
& e^{\int_{0}^{t} \sigma(\tau) d \tau}-\int_{0}^{\infty} Q(a, t) e^{\int_{0}^{t-a} \sigma(\tau) d \tau} d a \\
& =e^{\int_{0}^{t} \sigma(\tau) d \tau}\left(1-\int_{0}^{\infty} Q(a, t) e^{-\int_{t-a}^{t} \sigma(\tau) d \tau} d a\right) \leq 0 .
\end{aligned}
$$

Thus, to complete the proof, we will show that for a certain small positive constant $C$ and sufficiently large $j$ the following holds:

$$
C \int_{t-M}^{A_{m}} Q(a, t) e^{\int_{0}^{t-a} \sigma(\tau) d \tau} d a-K^{j+1} F(t) \leq 0
$$

By Lemma 2.2, $F(t) \geq \delta>0$ for $t \in\left(t_{1}, t_{2}\right)$. Let $a_{1}^{\prime}$ and $a_{2}^{\prime}$ be the same as in Corollary A.3. Then, according to (47), we have

$$
\rho_{1}(t) \geq K \rho_{0}(t)=K F(t)=\delta_{0}>0 \quad \text { for } t \in\left(a_{1}^{\prime}+t_{1}, a_{2}^{\prime}+t_{2}\right) .
$$

Iterating and using Corollary A.3 again, we obtain

$$
\rho_{j}(t) \geq K^{j} F(t) \geq \delta_{j}>0 \quad \text { for } t \in\left(a_{1}^{\prime}+j t_{1}, a_{2}^{\prime}+j t_{2}\right) .
$$

We choose $j$ such that $\left(M, M+A_{m}\right) \subset\left(a_{1}^{\prime}+(j+1) t_{1}, a_{2}^{\prime}+(j+1) t_{2}\right)$ for a sufficiently large $M$. Without loss of generality we can assume that it coincides with $M$ in the formulation of the theorem. By the choice of $j$, we have also

$$
K^{j+1} F(t)=\delta_{j+1}>0 \quad \text { for } t \in\left(a_{1}^{\prime}+(j+1) t_{1}, a_{2}^{\prime}+(j+1) t_{2}\right),
$$

which proves that the inequality (20) holds for a sufficiently small constant $C>0$.

We established that $\rho^{-}(t)$ is a lower solution to equation (6) for certain $j$ and $C$. For a small positive constant $C_{1}$ we have

$$
C_{1} e^{\int_{0}^{t} \sigma(\tau) d \tau} H(t-M) \leq \rho^{-}(t) \quad \text { for } t \geq M,
$$

and the inequality (18) follows.

Theorems 3.2 and 3.3 give us the following upper and lower bounds for the number of newborns. 
Corollary 3.4 If $\sigma \in L^{\infty}(0, \infty)$ satisfies equation (13), then there exist positive constants $C$ and $D$ such that

$$
C e^{\int_{0}^{t} \sigma(\tau) d \tau} \leq \rho(t) \leq D e^{\int_{0}^{t} \sigma(\tau) d \tau} \quad \text { for } t \geq M
$$

The total population $N(t)$ is defined by

$$
N(t)=\int_{0}^{\infty} n(a, t) d a, \quad t \geq 0 .
$$

It is of practical importance to study the large-time behavior not only of the function $\rho(t)$, but of the function $N(t)$ as well. According to Theorem 2.1, $N(t)$ can be written as

$$
N(t)=\int_{0}^{t} \rho(t-a) e^{-\int_{0}^{a} \mu(v, v+t-a) d v} d a+\int_{t}^{\infty} f(a-t) e^{-\int_{a-t}^{a} \mu(v, v+t-a) d v} d a .
$$

Combining Theorem 3.2 and Theorem 3.3 we obtain upper and lower bounds for the total population.

Theorem 3.5 If $\sigma \in L^{\infty}(0, \infty)$ satisfies equation (13) and $M>A_{\mu}$, then there exist two positive constants $C$ and $D$ such that

$$
C e^{\int_{0}^{t} \sigma(\tau) d \tau} \leq N(t) \leq D e^{\int_{0}^{t} \sigma(\tau) d \tau} \quad \text { for } t \geq M .
$$

Proof Suppose that $t \geq A_{\mu}$. Then, according to (ii)-(iii), we have

$$
\int_{t}^{\infty} f(a-t) e^{-\int_{a-t}^{a} \mu(v, v+t-a) d v} d a=\int_{0}^{A_{\mu}} f(a) e^{-\int_{a}^{a+t} \mu(v, v-a) d v} d a=0 .
$$

Therefore, because of (21),

$$
N(t)=\int_{0}^{t} \rho(t-a) e^{-\int_{0}^{a} \mu(v, v+t-a) d v} d a \quad \text { for } t \geq A_{\mu} .
$$

By Theorem 3.2, there exists a constant $D>0$ such that

$$
N(t) \leq D \int_{0}^{t} e^{\int_{0}^{t-a} \sigma(\tau) d \tau-\int_{0}^{a} \mu(v, v+t-a) d v} d a .
$$

Since $\sigma$ is bounded, we get the following upper bound for $N(t)$ :

$$
N(t) \leq D e^{\int_{0}^{t} \sigma(\tau) d \tau} \int_{0}^{A_{\mu}} e^{-\int_{t-a}^{t} \sigma(\tau) d \tau} d a \leq D_{1} e^{\int_{0}^{t} \sigma(\tau) d \tau} \quad \text { for } t \geq M
$$

To find a lower bound for $N(t)$, we use the fact that $\mu(a, t) \leq C_{\mu}$ for $a \in\left(0, a_{2}\right)$, and that $\sigma$ is a bounded function. According to Theorem 3.3 we obtain

$$
\begin{aligned}
N(t) & \geq C e^{\int_{0}^{t} \sigma(\tau) d \tau} \int_{0}^{t} e^{-\int_{t-a}^{t} \sigma(\tau) d \tau-\int_{0}^{a} \mu(v, v+t-a) d v} d a \\
& \geq C e^{\int_{0}^{t} \sigma(\tau) d \tau} \int_{0}^{a_{2}} e^{-\int_{t-a}^{t} \sigma(\tau) d \tau-C_{\mu} a_{2}} d a \geq C_{1} e^{\int_{0}^{t} \sigma(\tau) d \tau}
\end{aligned}
$$

for $t \geq M$. 


\section{Existence of the function $\sigma$}

Corollary 3.4 and Theorem 3.5 imply that, for large $t$, the functions $\rho(t)$ and $N(t)$ can be estimated using a function $\sigma \in L^{\infty}(0, \infty)$, which solves the characteristic equation (13). Until now, we assumed that such a function exists and in this section we provide a proof of its existence under the additional assumption that

$$
m(a, t)=0 \quad \text { for } 0<a<a_{m}
$$

where $a_{m}$ is a small number. We use $C_{\Lambda}^{0,1}(M, \infty)$, where $\Lambda>0$, to denote the Hölder space of all continuous functions $u$ defined on $[M, \infty)$ such that the norm

$$
\|u\|_{C_{\Lambda}^{0,1}(M, \infty)}=\|u\|_{L_{\Lambda}^{\infty}(M, \infty)}+\sup _{t_{1}, t_{2} \geq M} \frac{\left|u\left(t_{1}\right) e^{-\Lambda t_{1}}-u\left(t_{2}\right) e^{-\Lambda t_{2}}\right|}{\left|t_{1}-t_{2}\right|}
$$

is finite, where

$$
\|u\|_{L_{\Lambda}^{\infty}(M, \infty)}=\underset{t \geq M}{\operatorname{ess} \sup }|u(t)| e^{-\Lambda t} .
$$

In order to prove the main theorem of this section, we need the following lemma.

Lemma 4.1 Suppose that the function $Q$ is differentiable with respect to $t$ and $Q_{t}^{\prime}$ is bounded on $\mathbb{R}^{+} \times \mathbb{R}^{+}$. If $\gamma \in L^{\infty}(0, M)$, it satisfies

$$
\int_{0}^{\infty} Q(a, M) e^{-\int_{M-a}^{M} \gamma(\tau) d \tau} d a=1
$$

where $M \geq A_{m}$, then the integral equation

$$
h(t)=\int_{0}^{t-M} Q(a, t) h(t-a) d a+\int_{t-M}^{\infty} Q(a, t) e^{-\int_{t-a}^{M} \gamma(\tau) d \tau} d a, \quad t \geq M
$$

has a unique solution $h \in C_{\Lambda}^{0,1}(M, \infty)$ such that $h(M)=1$ and $h(t)>0$ for $t \geq M$ provided $\Lambda$ is sufficiently large.

Proof For $h \in C_{\Lambda}^{0,1}(M, \infty)$, we define the operator $H$ in the following way:

$$
H h(t)=T h(t)+g(t), \quad t \geq M,
$$

where

$$
\operatorname{Th}(t)=\int_{0}^{t-M} Q(a, t) h(t-a) d a, \quad t \geq M
$$

and

$$
g(t)=\int_{t-M}^{\infty} Q(a, t) e^{-\int_{t-a}^{M} \gamma(\tau) d \tau} d a, \quad t \geq M
$$

Due to the assumptions made, these integrals are continuous functions of $t$. 
We will prove that equation (24) has a unique solution using the fixed point argument. We begin by showing that $H$ maps $C_{\Lambda}^{0,1}(M, \infty)$ into $C_{\Lambda}^{0,1}(M, \infty)$. Let

$$
Q_{1}=\sup _{t \geq 0} \int_{0}^{\infty} Q(a, t) d a \quad \text { and } \quad Q_{2}=\sup _{t \geq 0} \int_{0}^{\infty} Q_{t}^{\prime}(a, t) d a
$$

and notice that $Q_{1}$ and $Q_{2}$ are finite. To estimate the left-hand side of (24), observe that for $h \in C_{\Lambda}^{0,1}(M, \infty)$, the following holds:

$$
\begin{aligned}
\|T h\|_{L_{\Lambda}^{\infty}(M, \infty)} & \leq \sup _{t \geq M} \int_{0}^{\infty} Q(a, t)|h(t-a)| e^{-\Lambda(t-a)} e^{-\Lambda a} d a \\
& \leq Q_{1} e^{-\Lambda a_{m}}\|h\|_{L_{\Lambda}^{\infty}(M, \infty)},
\end{aligned}
$$

where $a_{m}$ is defined by (22), and

$$
\begin{aligned}
\|g\|_{L_{\Lambda}^{\infty}(M, \infty)} & \leq \sup _{t \geq M} e^{-\Lambda t} \int_{0}^{\infty} Q(a, t) e^{-\int_{t-a}^{M} \gamma(\tau) d \tau} d a \\
& \leq Q_{1} e^{M\|\gamma\|_{L^{\infty}(0, M)}-\Lambda M} .
\end{aligned}
$$

Combining the previous inequalities, we arrive at

$$
\|H h\|_{L_{\Lambda}^{\infty}(M, \infty)} \leq\left(\|h\|_{L_{\Lambda}^{\infty}(M, \infty)}+e^{M\|\gamma\|_{L^{\infty}(0, M)}}\right) Q_{1} e^{-\Lambda M} .
$$

Let us assume that $t_{1}>t_{2}$. Then we have

$$
\begin{aligned}
& \left|T h\left(t_{1}\right) e^{-\Lambda t_{1}}-T h\left(t_{2}\right) e^{-\Lambda t_{2}}\right| \\
& =\mid \int_{0}^{t_{1}-M} Q\left(a, t_{1}\right) h\left(t_{1}-a\right) e^{-\Lambda t_{1}} d a-\int_{0}^{t_{2}-M} Q\left(a, t_{2}\right) h\left(t_{2}-a\right) e^{-\Lambda t_{2}} d a \\
& \quad-\int_{0}^{t_{2}-M} Q\left(a, t_{1}\right) h\left(t_{1}-a\right) e^{-\Lambda t_{1}} d a+\int_{0}^{t_{2}-M} Q\left(a, t_{1}\right) h\left(t_{1}-a\right) e^{-\Lambda t_{1}} d a \mid \\
& =\mid \int_{t_{2}-M}^{t_{1}-M} Q\left(a, t_{1}\right) h\left(t_{1}-a\right) e^{-\Lambda\left(t_{1}-a\right)} e^{-\Lambda a} d a \\
& \quad+\int_{0}^{t_{2}-M}\left(Q\left(a, t_{1}\right)-Q\left(a, t_{2}\right)\right) h\left(t_{2}-a\right) e^{-\Lambda\left(t_{2}-a\right)} e^{-\Lambda a} d a \\
& \quad+\int_{0}^{t_{2}-M} Q\left(a, t_{1}\right)\left(h\left(t_{1}-a\right) e^{-\Lambda\left(t_{1}-a\right)}-h\left(t_{2}-a\right) e^{-\Lambda\left(t_{2}-a\right)}\right) e^{-\Lambda a} d a \mid .
\end{aligned}
$$

This implies that

$$
\begin{aligned}
& \left|T h\left(t_{1}\right) e^{-\Lambda t_{1}}-T h\left(t_{2}\right) e^{-\Lambda t_{2}}\right| \\
& \quad \leq\left(Q_{1} e^{-\Lambda a_{m}}\|h\|_{L_{\Lambda}^{\infty}(M, \infty)}+\frac{Q_{2}}{\Lambda}\|h\|_{L_{\Lambda}^{\infty}(M, \infty)}+\frac{Q_{1}}{\Lambda}\|h\|_{C_{\Lambda}^{0,1}(M, \infty)}\right)\left|t_{1}-t_{2}\right|
\end{aligned}
$$

which leads us to the conclusion that

$$
\sup _{t_{1}, t_{2} \geq M} \frac{\left|T h\left(t_{1}\right) e^{-\Lambda t_{1}}-T h\left(t_{2}\right) e^{-\Lambda t_{2}}\right|}{\left|t_{1}-t_{2}\right|} \leq C\left(\frac{1}{\Lambda}+e^{-\Lambda a_{m}}\right)\|h\|_{C_{\Lambda}^{0,1}(M, \infty)} .
$$


Similarly, for the function $g(t)$, the following estimate holds:

$$
\left|g\left(t_{1}\right) e^{-\Lambda t_{1}}-g\left(t_{2}\right) e^{-\Lambda t_{2}}\right| \leq\left(Q_{1}+Q_{2}+\frac{Q_{1}}{\Lambda}\|\gamma\|_{L^{\infty}(0, M)}\right) e^{M\|\gamma\|_{L^{\infty}(0, M)}\left|t_{1}-t_{2}\right|},
$$

and from this we get

$$
\sup _{t_{1}, t_{2} \geq M} \frac{\left|g\left(t_{1}\right) e^{-\Lambda t_{1}}-g\left(t_{2}\right) e^{-\Lambda t_{2}}\right|}{\left|t_{1}-t_{2}\right|}<\infty .
$$

Combining the estimates above, we obtain $\|H h\|_{C_{\Lambda}^{0,1}(M, \infty)}<\infty$, which implies that $H$ maps $C_{\Lambda}^{0,1}(M, \infty)$ into $C_{\Lambda}^{0,1}(M, \infty)$.

Let now $h \in C_{\Lambda}^{0,1}(M, \infty)$. Estimates (25) and (26) yield

$$
\|T h\|_{C_{\Lambda}^{0,1}(M, \infty)} \leq C\left(\frac{1}{\Lambda}+e^{-\Lambda a_{m}}\right)\|h\|_{C_{\Lambda}^{0,1}(M, \infty)} .
$$

This and linearity of $T$ prove that the operator $H$ is a contraction on $C_{\Lambda}^{0,1}(M, \infty)$ for sufficiently large $\Lambda$. By the Banach fixed point theorem, equation (24) has a unique solution $h$.

The solution $h$ is a limit of the sequence $h_{n+1}=H h_{n}, h_{0}=0$ as $n \rightarrow \infty$. Its positivity follows from the fact that the sequence is monotonically increasing and each term is nonnegative on $[M, \infty)$.

Finally, for $t=M, H h(M)$ is equal to the left-hand side of (23), which completes the proof.

Theorem 4.2 Suppose that $Q$ is differentiable with respect to $t$ and $Q_{t}^{\prime}$ is bounded on $\mathbb{R}^{+} \times \mathbb{R}^{+}$. If $\gamma \in L^{\infty}(0, M)$, where $M \geq A_{m}$, satisfies (23), then the characteristic equation (13) has a unique solution $\sigma \in L^{\infty}(0, \infty)$ such that $\sigma=\gamma$ on $[0, M]$.

Proof Notice that equation (13) may be rewritten as

$$
\int_{0}^{t-M} Q(a, t) e^{\int_{M}^{t-a} \sigma(\tau) d \tau} d a+\int_{t-M}^{\infty} Q(a, t) e^{-\int_{t-a}^{M} \sigma(\tau) d \tau} d a=e^{\int_{M}^{t} \sigma(\tau) d \tau}
$$

where $t \geq M$. Multiplying both sides of the previous equation with $e^{\int_{0}^{M} \sigma(\tau) d \tau}$ leads us to

$$
\int_{0}^{t-M} Q(a, t) e^{\int_{0}^{t-a} \sigma(\tau) d \tau} d a+\int_{t-M}^{\infty} Q(a, t) e^{\int_{0}^{t-a} \sigma(\tau) d \tau} d a=e^{\int_{0}^{t} \sigma(\tau) d \tau} .
$$

Let $\sigma=\gamma$ on $[0, M]$, where $\gamma$ satisfies (23). Using the notation

$$
h(t)=e^{\int_{0}^{t} \sigma(\tau) d \tau},
$$

we obtain the equation

$$
h(t)=T h(t)+g(t)
$$

where

$$
\operatorname{Th}(t)=\int_{0}^{t-M} Q(a, t) e^{\int_{0}^{t-a} \sigma(\tau) d \tau} d a \quad \text { and } \quad g(t)=\int_{t-M}^{\infty} Q(a, t) e^{-\int_{t-a}^{M} \gamma(\tau) d \tau} d a
$$


for $t \geq M$. Since Th and $g$ belong to $C_{\Lambda}^{0,1}(M, \infty)$, Lemma 4.1 implies that equation (27) has a unique solution $h \in C_{\Lambda}^{0,1}(M, \infty)$. The function $\sigma$ is then defined for $t \geq M$ by

$$
\sigma(t)=\frac{h^{\prime}(t)}{h(t)}
$$

Using condition (23), we see that $h(M)=1$ and

$$
\int_{M}^{t} \frac{h^{\prime}(\tau)}{h(\tau)} d \tau=\ln h(t)-\ln h(M)=\ln h(t), \quad t \geq M
$$

which implies that $\sigma(t)$ satisfies (13).

\section{Upper and lower bounds through time-independent models}

According to Theorem 3.2, Theorem 3.3, and Theorem 3.5, the upper and lower bounds for the number of newborns and for the total population are functions of $\sigma$. Solving equation (13) to obtain $\sigma$ can be demanding and impractical. From the ecological point of view, it is often more important to know the trend that the population is following (i.e., whether it faces extinction or explosion) than the exact numbers of individuals at a certain point in time. This trade-off between precision and simplicity allows us to introduce time-independent best and worst case scenarios and to define $\sigma$ as the best and the worst constant intrinsic growth rate. To this end, we set

$$
Q_{+}(a)=\sup _{t \geq M} Q(a, t) \quad \text { and } \quad Q_{-}(a)=\inf _{t \geq M} Q(a, t), \quad a \geq 0
$$

when $M \geq A_{\mu}$. The functions $Q_{+}$and $Q_{-}$are nonnegative on $\mathbb{R}^{+}$and the integrals

$$
\int_{0}^{\infty} Q_{+}(a) e^{-k a} d a \text { and } \int_{0}^{\infty} Q_{-}(a) e^{-k a} d a
$$

are strictly monotonically decreasing functions of the parameter $k \in \mathbb{R}$. Therefore the equations

$$
\int_{0}^{\infty} Q_{+}(a) e^{-k a} d a=1 \text { and } \int_{0}^{\infty} Q_{-}(a) e^{-k a} d a=1
$$

have unique solutions $k_{+}$and $k_{-}$, respectively. Since

$$
\int_{0}^{\infty} Q(a, t) e^{-k_{-} a} d a \geq 1 \quad \text { and } \quad \int_{0}^{\infty} Q(a, t) e^{-k_{+} a} d a \leq 1 \quad \text { for } t \geq M
$$

direct application of Theorem 3.2 and Theorem 3.3 with $\sigma=k_{ \pm}$yields the next result.

Theorem 5.1 If $k_{+}$and $k_{-}$are defined by (28), then there exist positive constants $C$ and $D$ such that

$$
C e^{k_{-} t} \leq \rho(t) \leq D e^{k_{+} t} \quad \text { for } t \geq M
$$

Using Theorem 5.1, we obtain the estimates for the total population. 
Corollary 5.2 If $k_{+}$and $k_{-}$are defined by (28), then there exist positive constants Cand D such that

$$
C e^{k-t} \leq N(t) \leq D e^{k_{+} t} \quad \text { for } t \geq M
$$

Naturally, estimates of the number of newborns and the total population obtained by Theorem 3.2, Theorem 3.3, and Theorem 3.5 are finer than the estimates provided by Theorem 5.1 and Corollary 5.2, but they are harder to get. For practical purposes it is often enough to have a prognosis for population growth in the best and worst case, which makes the upper and lower bounds defined in Theorem 5.1 and Corollary 5.2 a useful tool for predicting the fate of a population.

\section{Periodical changes of the environment}

Quite often populations live in periodically changing habitats. Our general model allows any kind of temporal environmental change, but for practical reasons we study periodical changes in detail. We assume the birth rate is a periodic function with respect to time and the death rate is a time-independent function. Under these stronger conditions, we can find explicit forms of the upper and lower bounds for the number of newborns and for the total population.

It is worth noting that our results reveals a relation between the age-structure and the frequency of environmental changes. Namely, the generation time $T$, defined by

$$
T=\int_{0}^{\infty} Q(a) e^{-k_{0} a} a d a,
$$

represents the average age when individuals have their first offspring. Our next result casts some light on this relation.

Theorem 6.1 Let $\rho$ be a solution to equation (6) under the assumption that the birth rate satisfies

$$
m(a, t)=m(a)(1+\varepsilon \cos A(t-\gamma)), \quad a, t \geq 0
$$

and that $\mu$ only depends on $a$, where $A>0, \gamma \geq 0$, and $\varepsilon>0$ is a small number. In addition, let $k_{0}$ be a solution to equation

$$
\int_{0}^{\infty} Q(a) e^{-k_{0} a} d a=1
$$

where $Q(a)=m(a) e^{-\int_{0}^{a} \mu(v) d v}, a \geq 0$, and let $k_{2}$ be a solution to the equation

$$
k_{2}=\frac{1}{2 T}\left(\frac{1-\int_{0}^{\infty} Q(a) e^{-k_{0} a} \cos A a d a}{\left(\int_{0}^{\infty} Q(a) e^{-k_{0} a} \sin A a d a\right)^{2}+\left(1-\int_{0}^{\infty} Q(a) e^{-k_{0} a} \cos A a d a\right)^{2}}-1\right) .
$$

If

$$
\int_{0}^{\infty} Q(a) e^{\left(-k_{0}+i j A\right) a} d a \neq 1 \quad \text { for } j=1,2,
$$


then there exist positive constants $C, C_{1}$, and $C_{2}$ such that the inequality

$$
C_{1} e^{\left(k_{0}+k_{2} \varepsilon^{2}-C \varepsilon^{3}\right) t} \leq \rho(t) \leq C_{2} e^{\left(k_{0}+k_{2} \varepsilon^{2}+C \varepsilon^{3}\right) t}
$$

holds for sufficiently large $t$.

Proof According to Theorem 3.2 and Theorem 3.3, to prove the claim, it is sufficient to check that the inequalities

$$
\int_{0}^{\infty} Q(a) e^{-\int_{t-a}^{t} \sigma^{+}(\tau) d \tau} d a-\frac{1}{1+\varepsilon \cos A(t-\gamma)} \leq 0
$$

and

$$
\int_{0}^{\infty} Q(a) e^{-\int_{t-a}^{t} \sigma^{-}(\tau) d \tau} d a-\frac{1}{1+\varepsilon \cos A(t-\gamma)} \geq 0,
$$

hold for sufficiently large $t$, where the functions $\sigma^{ \pm}(t)$ are defined by

$$
\begin{aligned}
\sigma^{ \pm}(t)= & k_{0}+\varepsilon\left(p_{1} \sin A t+q_{1} \cos A t\right) \\
& +\varepsilon^{2}\left(k_{2}+p_{2} \sin 2 A t+q_{2} \cos 2 A t\right) \pm C \varepsilon^{3},
\end{aligned}
$$

and $C$ is a positive constant; $p_{1}, p_{2}, q_{1}$, and $q_{2}$ are constants to be defined later. For brevity, we write

$$
\sigma^{ \pm}(t)=k_{0}+\varepsilon \sigma_{1}(t)+\varepsilon^{2} \sigma_{2}(t) \pm C \varepsilon^{3}
$$

where

$$
\sigma_{1}(t)=p_{1} \sin A t+q_{1} \cos A t
$$

and

$$
\sigma_{2}(t)=k_{2}+p_{2} \sin 2 A t+q_{2} \cos 2 A t .
$$

We have

$$
\begin{aligned}
\int_{0}^{\infty} & Q(a) e^{-\int_{t-a}^{t} \sigma^{+}(\tau) d \tau} d a-\frac{1}{1+\varepsilon \cos A(t-\gamma)} \\
= & \int_{0}^{\infty} Q(a) e^{-\int_{t-a}^{t}\left(k_{0}+\varepsilon \sigma_{1}(\tau)+\varepsilon^{2} \sigma_{2}(\tau)+C \varepsilon^{3}\right) d \tau} d a-\frac{1}{1+\varepsilon \cos A(t-\gamma)} \\
= & \int_{0}^{\infty} Q(a) e^{-k_{0} a}\left(1-\varepsilon \int_{t-a}^{t} \sigma_{1}(\tau) d \tau-\varepsilon^{2} \int_{t-a}^{t} \sigma_{2}(\tau) d \tau-C \varepsilon^{3} a\right. \\
& +\frac{\varepsilon^{2}}{2}\left(\int_{t-a}^{t} \sigma_{1}(\tau) d \tau\right)^{2}+\varepsilon^{3} \int_{t-a}^{t} \sigma_{1}(\tau) d \tau \int_{t-a}^{t} \sigma_{2}(\tau) d \tau \\
& \left.-\frac{\varepsilon^{3}}{6}\left(\int_{t-a}^{t} \sigma_{1}(\tau) d \tau\right)^{3}+O\left(\varepsilon^{4}\right)\right) d a \\
& -\left(1-\varepsilon \cos A(t-\gamma)+\varepsilon^{2} \cos ^{2} A(t-\gamma)+O\left(\varepsilon^{4}\right)\right) .
\end{aligned}
$$


Collecting the terms with the same power of $\varepsilon$ and noting that $Q(a)=0$ outside of the interval $\left(a_{m}, A_{m}\right)$, we obtain

$$
\begin{aligned}
\int_{0}^{\infty} Q(a) e^{-\int_{t-a}^{t} \sigma^{+}(\tau) d \tau} d a-\frac{1}{1+\varepsilon \cos A(t-\gamma)} \\
=\int_{0}^{\infty} Q(a) e^{-k_{0} a} d a-1 \\
\quad-\varepsilon\left(\int_{0}^{\infty} Q(a) e^{-k_{0} a} \int_{t-a}^{t} \sigma_{1}(\tau) d \tau d a-\cos A(t-\gamma)\right) \\
\quad+\varepsilon^{2}\left(\int_{0}^{\infty} Q(a) e^{-k_{0} a}\left(\frac{1}{2}\left(\int_{t-a}^{t} \sigma_{1}(\tau) d \tau\right)^{2}-\int_{t-a}^{t} \sigma_{2}(\tau) d \tau\right) d a\right. \\
\left.\quad-\cos ^{2} A(t-\gamma)\right) \\
\quad-\varepsilon^{3}\left(\int _ { 0 } ^ { \infty } Q ( a ) e ^ { - k _ { 0 } a } \left(\mathrm{Ca}-\int_{t-a}^{t} \sigma_{1}(\tau) d \tau \int_{t-a}^{t} \sigma_{2}(\tau) d \tau\right.\right. \\
\left.\left.\quad+\frac{1}{6}\left(\int_{t-a}^{t} \sigma_{1}(\tau) d \tau\right)^{3}\right) d a\right)+O\left(\varepsilon^{4}\right) .
\end{aligned}
$$

Due to equation (31), the term (35) is equal to zero.

In the next step we will show that the term (36) is zero. Observe that $e^{-k_{0} a}$ is bounded and that function $Q(a)$ has compact support according to assumption (i). We use the notation

$$
\phi(a)=\int_{a}^{\infty} Q(x) e^{-k_{0} x} d x, \quad a \geq 0
$$

and from (36) we obtain the equation

$$
\int_{0}^{\infty} \phi^{\prime}(a) \int_{t-a}^{t} \sigma_{1}(\tau) d \tau d a=-\cos A(t-\gamma)
$$

Solving this equation leads to

$$
\int_{0}^{\infty} \phi(a)\left(p_{1} \sin A(t-a)+q_{1} \cos A(t-a)\right) d a=-\cos A(t-\gamma),
$$

and therefore

$$
\int_{0}^{\infty} \phi(a)\left(p_{1} \cos A a+q_{1} \sin A a\right) d a=-\sin A \gamma
$$

and

$$
\int_{0}^{\infty} \phi(a)\left(q_{1} \cos A a-p_{1} \sin A a\right) d a=-\cos A \gamma .
$$

The last two equations are equivalent to

$$
\int_{0}^{\infty} \phi(a)\left(\begin{array}{cc}
\cos A a & \sin A a \\
-\sin A a & \cos A a
\end{array}\right)\left(\begin{array}{l}
p_{1} \\
q_{1}
\end{array}\right) d a=\left(\begin{array}{l}
-\sin A \gamma \\
-\cos A \gamma
\end{array}\right) .
$$


We use $I_{1}(A)$ and $I_{2}(A)$ to denote

$$
I_{1}(A)=\int_{0}^{\infty} \phi(a) \cos A a d a \text { and } I_{2}(A)=\int_{0}^{\infty} \phi(a) \sin A a d a,
$$

respectively. After a partial integration, we have

$$
I_{1}(A)=\frac{1}{A} \int_{0}^{\infty} Q(a) e^{-k_{0} a} \sin A a d a
$$

and

$$
I_{2}(A)=\frac{1}{A}\left(1-\int_{0}^{\infty} Q(a) e^{-k_{0} a} \cos A a d a\right) .
$$

The assumption $\int_{0}^{\infty} Q(a) e^{\left(-k_{0}+i A\right) a} d a \neq 1$ implies that $I_{1}^{2}(A)+I_{2}^{2}(A) \neq 0$. Therefore the system of equations (41) has the unique solution given by

$$
\left(\begin{array}{l}
p_{1} \\
q_{1}
\end{array}\right)=\frac{1}{I_{1}^{2}(A)+I_{2}^{2}(A)}\left(\begin{array}{cc}
I_{1}(A) & -I_{2}(A) \\
I_{2}(A) & I_{1}(A)
\end{array}\right)\left(\begin{array}{l}
-\sin A \gamma \\
-\cos A \gamma
\end{array}\right),
$$

and this solution satisfies

$$
p_{1}^{2}+q_{1}^{2}=\frac{1}{I_{1}^{2}(A)+I_{2}^{2}(A)}
$$

This proves that the term (36) is zero if $p_{1}$ and $q_{1}$ are defined by (42).

To prove that the term (37) is zero, we solve the following equation:

$$
-\int_{0}^{\infty} \phi^{\prime}(a)\left(\frac{1}{2}\left(\int_{t-a}^{t} \sigma_{1}(\tau) d \tau\right)^{2}-\int_{t-a}^{t} \sigma_{2}(\tau) d \tau\right) d a=\frac{1+\cos 2 A(t-\gamma)}{2} .
$$

This is equivalent to

$$
\begin{aligned}
\int_{0}^{\infty} \phi^{\prime}(a) \int_{t-a}^{t} \sigma_{2}(\tau) d \tau d a= & \frac{1+\cos 2 A(t-\gamma)}{2} \\
& +\frac{1}{2} \int_{0}^{\infty} \phi^{\prime}(a)\left(\int_{t-a}^{t} \sigma_{1}(\tau) d \tau\right)^{2} d a
\end{aligned}
$$

To compute the integral in the right-hand side of (44) observe that

$$
\begin{aligned}
\int_{t-a}^{t} \sigma_{1}(\tau) d \tau= & \frac{1}{A}\left(-p_{1}+p_{1} \cos A a+q_{1} \sin A a\right) \cos A t \\
& +\frac{1}{A}\left(p_{1} \sin A a+q_{1}-q_{1} \cos A a\right) \sin A t
\end{aligned}
$$

and

$$
\frac{A}{2 \pi} \int_{0}^{2 \pi / A}\left(\int_{t-a}^{t} \sigma_{1}(\tau) d \tau\right)^{2} d t=\frac{p_{1}^{2}+q_{1}^{2}}{A^{2}}(1-\cos A a) .
$$


Integrating equation (44) and using (45), we get

$$
\begin{aligned}
\frac{A}{2 \pi} & \int_{0}^{2 \pi / A} \int_{0}^{\infty} \phi^{\prime}(a) \int_{t-a}^{t} \sigma_{2}(\tau) d \tau d a d t \\
= & \frac{A}{2 \pi} \int_{0}^{2 \pi / A} \frac{1+\cos 2 A(t-\gamma)}{2} d t \\
& +\frac{1}{2} \int_{0}^{\infty} \phi^{\prime}(a) \frac{A}{2 \pi} \int_{0}^{2 \pi / A}\left(\int_{t-a}^{t} \sigma_{1}(\tau) d \tau\right)^{2} d t d a \\
= & \frac{1}{2}+\frac{p_{1}^{2}+q_{1}^{2}}{2 A^{2}} \int_{0}^{\infty} \phi^{\prime}(a)(1-\cos A a) d a,
\end{aligned}
$$

which implies that

$$
k_{2} \int_{0}^{\infty} \phi^{\prime}(a) a d a=\frac{1}{2}+\frac{p_{1}^{2}+q_{1}^{2}}{2 A^{2}} \int_{0}^{\infty} \phi^{\prime}(a)(1-\cos A a) d a .
$$

By (43), this becomes

$$
k_{2} \int_{0}^{\infty} \phi^{\prime}(a) a d a=\frac{1}{2}\left(1+\frac{1}{A^{2}\left(I_{1}^{2}(A)+I_{2}^{2}(A)\right)} \int_{0}^{\infty} \phi^{\prime}(a)(1-\cos A a) d a\right) .
$$

According to (39), we get

$$
\int_{0}^{\infty} \phi^{\prime}(a) a d a=-\int_{0}^{\infty} Q(a) e^{-k_{0} a} a d a,
$$

and according to (35) and (37),

$$
\int_{0}^{\infty} \phi^{\prime}(a)(1-\cos A a) d a=-1+\int_{0}^{\infty} Q(a) e^{-k_{0} a} \cos A a d a .
$$

From this it follows that the parameter $k_{2}$ is given by (32).

Equation (44) implies that $p_{2}$ and $q_{2}$ can be obtained by solving

$$
\int_{0}^{\infty} \phi^{\prime}(a) \int_{t-a}^{t}\left(p_{2} \sin 2 A \tau+q_{2} \cos 2 a \tau\right) d \tau d a=\frac{\cos 2 A(t-\gamma)}{2} .
$$

Using reasoning similar to the one by which we solved (40) to obtain $p_{1}$ and $q_{1}$, we see that $p_{2}$ and $q_{2}$ are given by

$$
\left(\begin{array}{l}
p_{2} \\
q_{2}
\end{array}\right)=\frac{1}{I_{1}^{2}(2 A)+I_{2}^{2}(2 A)}\left(\begin{array}{cc}
I_{1}(2 A) & -I_{2}(2 A) \\
I_{2}(2 A) & I_{1}(2 A)
\end{array}\right)\left(\begin{array}{c}
\frac{\sin A \gamma}{2} \\
\frac{\cos A \gamma}{2}
\end{array}\right) .
$$

Notice that according to the assumption of the theorem, $I_{1}^{2}(2 A)+I_{2}^{2}(2 A) \neq 0$, and the solutions to (46) exist. This proves that the term (37) is zero if the constants $k_{2}, p_{2}$, and $q_{2}$ are given by (32) and (46).

It is clear now that (34) is negative only if (38) is negative. Since $\int_{t-a}^{t} \sigma_{1}(\tau) d \tau$ and $\int_{t-a}^{t} \sigma_{2}(\tau) d \tau$ are bounded functions for all $t$, it follows that the term (34) is negative provided that $C$ is sufficiently large. 
From this, by Theorem 3.2, it follows that

$$
\rho(t) \leq c_{2} e^{\left(k_{0}+\varepsilon^{2} k_{2}+C \varepsilon^{3}\right) t+g(t)} \quad \text { for } t \geq M
$$

where

$$
g(t)=\frac{1}{A}\left(p_{1}-p_{1} \cos A t+q_{1} \sin A t\right)+\frac{1}{2 A}\left(p_{2}-p_{2} \cos 2 A t+q_{2} \sin 2 A t\right), \quad t \geq 0,
$$

is a bounded function. Finally, for $C_{2}=c_{2} \max _{t \geq 0} e^{g(t)}$, we obtain the right-hand side of inequality (33).

In a similar way we prove the left-hand side inequality of (33).

Corollary 6.2 Under the assumptions of Theorem 6.1, there exist positive constants $C, D_{1}$, and $D_{2}$ such that the total population $N(t)$ can be estimated as follows:

$$
D_{1} e^{\left(k_{0}+\varepsilon^{2} k_{2}-C \varepsilon^{3}\right) t} \leq N(t) \leq D_{2} e^{\left(k_{0}+\varepsilon^{2} k_{2}+C \varepsilon^{3}\right) t}
$$

for sufficiently large $t$.

The behavior of population growth in cyclic environments for discrete time has already been analyzed. As expected, our results correspond to the main results presented by Tuljapurkar in [15]. According to Corollary 6.2, and due to the fact that $\varepsilon>0$ is a small number, the large-time behavior of $N(t)$ is determined by the parameter $k_{0}$. For negative values of $k_{0}$, the total population is decreasing and extinction of the population is imminent. On the other hand, a positive $k_{0}$ yields population growth and survival of population is granted. In the borderline case when $k_{0}=0$, the behavior of the total population is determined by the parameter $k_{2}$. We observe population growth for positive $k_{2}$ and population decline for negative $k_{2}$.

These considerations can be reformulated in the terms of the net reproductive rate and transient vital rates. The net reproductive rate (or the average number of offspring per individual) is defined by

$$
R_{0}=\int_{0}^{\infty} Q(a) d a .
$$

Comparing the expression for $R_{0}$ with the expression for $k_{0}$, given by (31), we come to the conclusion that for large $t$ the following hold:

(1) If $R_{0}<1$, then $k_{0}<0$ and $N(t) \rightarrow 0$ as $t \rightarrow \infty$ for small $\varepsilon$.

(2) If $R_{0}>1$, then $k_{0}>0$ and $N(t) \rightarrow \infty$ as $t \rightarrow \infty$ for small $\varepsilon$.

(3) If $R_{0}=1$, then $k_{0}=0$ and $N(t) \rightarrow \infty$ or $N(t) \rightarrow 0$ as $t \rightarrow \infty$, depending on the sign of $k_{2}$.

The average value $\sigma_{\mathrm{av}}$ of the function $\sigma(t)$ over a period is defined by

$$
\sigma_{\mathrm{av}}=\frac{A}{2 \pi} \int_{0}^{2 \pi / A} \sigma(\tau) d \tau .
$$

In the light of Theorem 6.1, it satisfies

$$
\sigma_{\mathrm{av}}=k_{0}+\varepsilon^{2} k_{2}+O\left(\varepsilon^{3}\right)
$$


implying that the average number of newborns is

$$
\rho_{\mathrm{av}}(t)=C e^{\left(k_{0}+\varepsilon^{2} k_{2}+O\left(\varepsilon^{3}\right)\right) t} .
$$

Consequently, the average total population is

$$
N_{\mathrm{av}}(t)=D e^{\left(k_{0}+\varepsilon^{2} k_{2}+O\left(\varepsilon^{3}\right)\right) t},
$$

where $C$ and $D$ are positive constants. As expected, the conclusion in terms of averages is analogous to what we have already mentioned. For negative $k_{0}$, the average population is going to extinction; for $k_{0}=0$, the average population is constant; for positive $k_{0}$, the average population is growing.

\section{Improvement of stability due to a variable environment}

According to equation (32), for fixed birth and death rates, the parameter $k_{2}$ can change its sign for different values of $A$. By Theorem 6.1 and Corollary 6.2 it is obvious that changes in $k_{2}$ reflect on population growth either by promoting it (for $k_{2}>0$ ) or by dampening it (for $k_{2}<0$ ). In order to get some insight into the behavior of the parameter $k_{2}$ and its effect on the persistence of the population, we use the real life data for the vital rates for four different species. Although $k_{2}$ depends not only on the frequency of oscillation, but also on the vital rates, here we will focus on changes in the frequency.

Table 1 contains the mean data for four typical life histories. The ursus life history represents a long-lived species with high survival rate and low birth rate. The calidris has

\begin{tabular}{|c|c|c|c|c|c|c|c|c|}
\hline \multirow[t]{2}{*}{ Age class } & \multicolumn{2}{|c|}{ Ursus } & \multicolumn{2}{|c|}{ Calidris } & \multicolumn{2}{|c|}{ Ectotherm } & \multicolumn{2}{|c|}{ Insect } \\
\hline & $s$ & $m$ & $s$ & $m$ & $s$ & $m$ & $s$ & $m$ \\
\hline 1 & 0.67 & 0 & 0.32 & 0 & 0.13 & 0 & 0.54 & 0 \\
\hline 2 & 0.75 & 0 & 0.78 & 1 & 0.2 & 1 & 0.52 & 0 \\
\hline 3 & 0.82 & 0 & 0.72 & 1 & 0.17 & 30 & 0.49 & 0 \\
\hline 4 & 0.9 & 0.5 & 0.66 & 1 & 0.14 & 30 & 0.47 & 0 \\
\hline 5 & 0.86 & 0.5 & 0.6 & 1 & 0.11 & 30 & 0.45 & 0 \\
\hline 6 & 0.82 & 0.5 & 0.54 & 1 & 0.09 & 30 & 0.42 & 0 \\
\hline 7 & 0.78 & 0.5 & 0.48 & 1 & 0.06 & 30 & 0.4 & 0 \\
\hline 8 & 0.74 & 0.5 & 0.42 & 1 & 0.03 & 30 & 0.38 & 83.33 \\
\hline 9 & 0.7 & 0.5 & 0.36 & 1 & 0.01 & 30 & 0.36 & 166.67 \\
\hline 10 & 0.65 & 0.5 & 0.3 & 1 & 0 & 30 & 0.34 & 250 \\
\hline 11 & 0.61 & 0.5 & 0.24 & 1 & & & 0.32 & 333.33 \\
\hline 12 & 0.57 & 0.5 & 0.18 & 1 & & & 0.3 & 416.67 \\
\hline 13 & 0.53 & 0.5 & 0.12 & 1 & & & 0.27 & 500 \\
\hline 14 & 0.49 & 0.5 & 0.06 & 1 & & & 0.25 & 433.33 \\
\hline 15 & 0.45 & 0.5 & 0.01 & 1 & & & 0.23 & 400 \\
\hline 16 & 0.41 & 0.5 & 0 & 1 & & & 0.21 & 366.67 \\
\hline 17 & 0.37 & 0.5 & & & & & 0.19 & 333.33 \\
\hline 18 & 0.33 & 0.5 & & & & & 0.17 & 300 \\
\hline 19 & 0.29 & 0.5 & & & & & 0.15 & 266.67 \\
\hline 20 & 0.25 & 0.5 & & & & & 0.13 & 233.33 \\
\hline 21 & 0.21 & 0.5 & & & & & 0.11 & 200 \\
\hline 22 & 0.16 & 0.5 & & & & & 0.09 & 166.67 \\
\hline 23 & 0.12 & 0.5 & & & & & 0.06 & 133.33 \\
\hline 24 & 0.08 & 0.5 & & & & & 0.04 & 100 \\
\hline 25 & 0.04 & 0.5 & & & & & 0.02 & 66.67 \\
\hline 26 & 0.01 & 0.5 & & & & & 0.01 & 33.33 \\
\hline 27 & 0 & 0.5 & & & & & & \\
\hline
\end{tabular}

Table 1 Life histories 


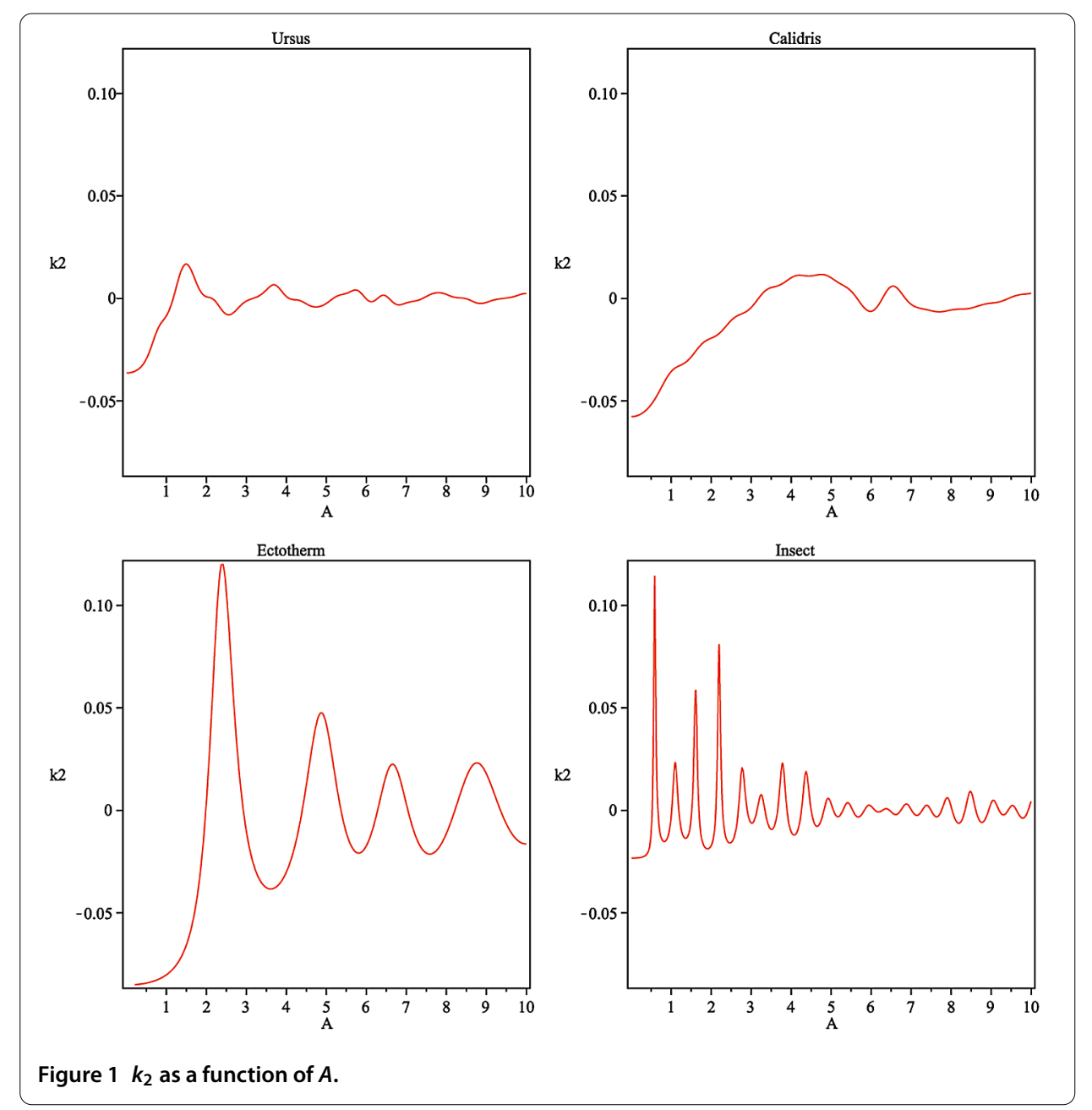

shorter life and low survival and low birth rate. The ectotherm has an even shorter life than ursus and calidris, a lower survival rate, and a higher birth rate. The insect has a very short life - age classes are given in weeks, while for the other three representatives age classes are given in years. The survival rate for the insect is very low, but the birth rate is extremely high.

The vital rates in Table 1 are the birth rate $m$ and the survival probability $s$, given by $s(a)=e^{-\int_{a-1}^{a} \mu(v) d v}$ for $a \in\left[1, A_{\mu}\right]$. We define the periodic birth rate using (30). For simplicity, we assume that $\gamma=0$.

We use equation (31) to compute $k_{0}$ for all species and (32) to plot graphs of the function $k_{2}(A)$ for the frequencies $A \in[0.01,10]$ for all four life histories.

According to Figure $1, k_{2}(A)<0$ for small values of $A$ in all cases and it changes its sign for different values of $A$. The dominant value of $k_{2}(A)$ is obtained for $A$ comparable to $A_{T}$, where $A_{T}$ is the frequency of oscillation corresponding to the generation time $T$ defined by (29).

By Theorem 6.1 and Corollary 6.2, the upper and the lower bounds for the number of newborns and the total population are depends on $k_{2}$. Therefore, oscillations with periods much longer than the generation time yield negative $k_{2}(A)$ for all species. In these cases population growth and chance of survival are decreased by environmental change. Os- 
Table 2 Characteristic parameters

\begin{tabular}{lllll}
\hline & Ursus & Calidris & Ectotherm & Insect \\
\hline$k_{0}$ & -0.0409 & -0.1234 & -0.6569 & -0.1214 \\
$R_{0}$ & 0.78 & 0.6874 & 0.1789 & 0.3529 \\
$A_{T}$ & 1.36 & 1.95 & 2.28 & 0.59 \\
$T$ & 4.59 & 3.21 & 2.75 & 10.54 \\
\hline
\end{tabular}

cillations with a period comparable to the generation time need a more detailed analysis. Namely, from Figure 1, and Table 2, we see that oscillations with periods slightly longer than generation time are detrimental for ursus and calidris, but they are beneficial for ectotherm and insect. Conversely, oscillations with periods slightly shorter than the generation time are beneficial for ursus and calidris, but they are detrimental for ectotherm and insect.

We would like to point out that our conclusions are valid for the vital rates given in Table 1. From equation (32) it follows that $k_{2}$ is a function of the generation time $T$. Since the generation time $T$ depends on life history, it is worth investigating how changes in life history will reflect on the population growth in a periodic environment.

\section{Discussion}

We studied a population model derived from the linear age-structured time-dependent model presented by Chipot in [1]. The difference between these two models is in the properties of the vital rates. From the biological point of view, this approach is justified since we need a tool for analyzing population growth and predicting extinctions or explosions of population due to temporally changing environment. We obtained several new results which are in accordance with the classical theory developed for the time-independent or for the discrete models.

Contrary to the Lotka-Euler characteristic equation (12) in the classical time-independent model, the characteristic equation in the time-dependent model is given by (13), which emphasizes the fact that we study the interplay between age- and time-dependent vital rates and population growth.

The theory we developed established that if $\sigma(t)$ is a solution to the characteristic equation, then both the number of newborns, $\rho(t)$, and the total population, $N(t)$, belong to a certain region whose upper and lower boundaries depend on $\sigma(t)$. Namely, we proved that

$$
C_{1} e^{\int_{0}^{t} \sigma(\tau) d \tau} \leq \rho(t) \leq D_{1} e^{\int_{0}^{t} \sigma(\tau) d \tau}
$$

and

$$
C_{2} e^{\int_{0}^{t} \sigma(\tau) d \tau} \leq N(t) \leq D_{2} e^{\int_{0}^{t} \sigma(\tau) d \tau}
$$

for sufficiently large $t$ and some positive constants $C_{1}, C_{2}, D_{1}$, and $D_{2}$.

Solving integral equation (13) in order to find $\sigma(t)$ is usually a difficult task. Therefore we use upper and lower solutions and instead of dealing with the original problem, we formulate and analyze the worst and the best case scenarios. These simplified problems are time-independent and for them we have the following result:

$$
C e^{k_{-} t} \leq N(t) \leq D e^{k_{+} t} \quad \text { for large } t
$$


where $C, D>0$ and the parameters $k_{ \pm}$are solutions to the best and worst case characteristic equations. This means that for large $t$, the total population can be estimated either by $e^{\int_{0}^{t} \sigma(\tau) d \tau}$ or by its worst and best case counterparts $e^{k \pm t}$. Estimates that include the function $\sigma(t)$ are finer in comparison with the ones obtained by $k_{ \pm}$, but they also require solving the characteristic equation (13). The loss in precision is small enough to justify the use of the upper and lower bounds $e^{k_{ \pm} t}$ for determining the large-time behavior of the functions $\rho(t)$ and $N(t)$.

For populations that live in a periodically changing environment we can be more precise. In addition to the general results, in the periodic case we have explicit formulas for the upper and lower boundaries. We assumed the birth rate is a periodic function with respect to time and the death rate is only age dependent. For sufficiently large $t$, we proved that

$$
C_{1} e^{\left(k_{0}+\varepsilon^{2} k_{2}-\alpha \varepsilon^{3}\right) t} \leq \rho(t) \leq D_{1} e^{\left(k_{0}+\varepsilon^{2} k_{2}+\alpha \varepsilon^{3}\right) t}
$$

and

$$
C_{2} e^{\left(k_{0}+\varepsilon^{2} k_{2}-\alpha \varepsilon^{3}\right) t} \leq N(t) \leq D_{2} e^{\left(k_{0}+\varepsilon^{2} k_{2}+\alpha \varepsilon^{3}\right) t},
$$

where $C_{i}, D_{i}, i=1,2$, and $\alpha$ are positive constants and $\varepsilon>0$ is a small number. We proved that the parameter $k_{0}$ depends only on the vital rates, while the parameter $k_{2}$ depends on the vital rates and on the frequency $A$ of oscillation of the birth rate.

Since $\varepsilon$ is a small number, the dominant role in the asymptotic behavior of the total population $N(t)$ belongs to the parameter $k_{0}$. For $k_{0}>0$, the population is growing, while for $k_{0}<0$ it is declining. In the special case when $k_{0}=0$, the large-time behavior of the population is entirely determined by the sign of $k_{2}(A)$, which is, according to (32), a function depending on the frequency $A$.

Using real life data we came to the following conclusions. If the period of oscillation is much longer than the generation time, the oscillation decreases the growth of a population in all observed cases. On the other hand, with oscillations whose periods are comparable to the generation time, we must be more careful. If the period is shorter than the generation time, changes in the environment increase the population growth for ursus and calidris and reduce it for ectotherm and insect. However, if the period is longer that the generation time, populations of ursus and calidris will decrease and the populations of ectotherm and insect will increase.

Our results expand those from a discrete model in Tuljapurkar [15]. The differences are in the fact that he deals with the discrete population model and does not use different life histories. Moreover, he claims that oscillations with period either much longer or shorter that the generation time are detrimental for population growth, and oscillations with period comparable to the generation time are beneficial for population growth. We show that for periods comparable to the generation time, there does not exist an unanimous effect on population growth for all species and all period lengths.

Finally, according to (32), $k_{2}$ is a function of the vital rates and frequency. It is worth investigating how changes in life history reflect on the behavior of the function $k_{2}$.

\section{Appendix}

Proof of Theorem 2.1 Assume that $n$ is a solution to problem (1)-(3). Using the change of variables $a=x, t=x+y$, we transform the partial differential equation (1) into an ordinary 
differential equation with respect to $x$ :

$$
\frac{d n(x, x+y)}{d x}=\frac{\partial n(a, t)}{\partial t}+\frac{\partial n(a, t)}{\partial a}=-\mu(x, x+y) n(x, x+y) .
$$

For $y>0$, the solution to this equation is

$$
n(x, x+y)=n(0, y) e^{-\int_{0}^{x} \mu(v, v+y) d v}
$$

and shifting back to $a$ and $t$ yields

$$
n(a, t)=n(0, t-a) e^{-\int_{0}^{a} \mu(v, v+t-a) d v} \quad \text { for } t>a .
$$

For $y \leq 0$, the solution is given by

$$
n(x, x+y)=n(x, 0) e^{-\int_{-y}^{x} \mu(v, v+y) d v},
$$

and since $n(a, 0)=f(a)$, we obtain

$$
n(a, t)=f(a-t) e^{-\int_{a-t}^{a} \mu(v, v+t-a) d v} \quad \text { for } t \leq a .
$$

To prove that $\rho(t)=n(0, t)$ satisfies equation (6), observe that, according to (2),

$$
\begin{aligned}
\rho(t)= & \int_{0}^{t} m(a, t) n(a, t) d a+\int_{t}^{\infty} m(a, t) n(a, t) d a \\
= & \int_{0}^{t} m(a, t) \rho(t-a) e^{-\int_{0}^{a} \mu(v, v+t-a) d v} d a \\
& +\int_{t}^{\infty} m(a, t) f(a-t) e^{-\int_{a-t}^{a} \mu(v, v+t-a) d v} d a .
\end{aligned}
$$

Making use of the following corollary of the Banach fixed point theorem, we examine the behavior of the solutions of equation (11). For the proof and details, see Chapter 7 in [16].

Lemma A.1 Suppose that $X$ is a Banach space, $M$ is a closed nonempty subset of $X$, and $P$ is a metric space with metric borrowed from $L^{\infty}\left(\mathbb{R}^{n} \times \mathbb{R}^{n}\right)$. Suppose, moreover, that $T_{p}$ is a contraction on $M$, for every $p \in P$, with contraction constant independent of $p$, such that $T_{p} x \rightarrow T_{p_{0}} x$ in $X$ as $p \rightarrow p_{0}$ for every $x \in X$ and every $p \in P$. Then $T_{p}$ has a unique fixed point $x_{p} \in M$ for every $p \in P$ and $x_{p} \rightarrow x_{p_{0}}$ as $p \rightarrow p_{0}$.

Proof of Theorem 2.7 If $m \in P$, then, according to Lemma 2.3, the operator $K_{m}$ is a contraction on $L_{\Lambda}^{\infty}(0, \infty)$ for sufficiently large $\Lambda$. The contraction constant $k=Q^{*} / \Lambda$, defined in (11), can be chosen independently of $m$. For fixed $m_{0} \in P$, we have

$$
\begin{aligned}
& \left\|K_{m} \rho+F_{m}-K_{m_{0}} \rho-F_{m_{0}}\right\|_{\Lambda} \\
& \quad \leq \sup _{t \geq 0} \int_{0}^{t}\left|m(a, t)-m_{0}(a, t)\right| \rho(t-a) e^{-\Lambda(t-a)} e^{-\Lambda a} d a
\end{aligned}
$$




$$
\begin{aligned}
&+\sup _{t \geq 0} e^{-\Lambda t} \int_{t}^{\infty}\left|m(a, t)-m_{0}(a, t)\right| f(a-t) d a \\
& \leq\left\|m-m_{0}\right\|_{L^{\infty}\left(\mathbb{R}^{+} \times \mathbb{R}^{+}\right)}\left(\frac{\|\rho\|_{\Lambda}}{\Lambda}+\|f\|_{L^{1}\left(\mathbb{R}^{+}\right)}\right) .
\end{aligned}
$$

Hence all conditions of Lemma A.1 are satisfied and the conclusion follows.

For proving Theorem 3.3, we need the following lemma.

Lemma A.2 Let $f$ and $g$ be nonnegative measurable functions such that $f(t) \geq \theta_{1}>0$ for $t \in\left(t_{1}, t_{2}\right)$ and $g(a, t) \geq \theta_{2}>0$ for all $t \geq 0$ when $a \in\left(a_{1}, a_{2}\right)$. Then

$$
\int_{0}^{t} g(a, t) f(t-a) d a \geq \theta_{1} \theta_{2} \min \left(t_{2}-t_{1}, a_{2}-a_{1}, t-t_{1}-a_{1}, a_{2}+t_{2}-t\right)>0
$$

for $t \in\left(t_{1}+a_{1}, t_{2}+a_{2}\right)$.

Proof Let $t \in\left(t_{1}+a_{1}, t_{2}+a_{2}\right)$. By the assumptions we have

$$
\begin{aligned}
\int_{0}^{t} g(a, t) f(t-a) d a & \geq \theta_{2} \int_{a_{1}}^{\min \left(t, a_{2}\right)} f(t-a) d a=\theta_{2} \int_{\max \left(0, t-a_{2}\right)}^{t-a_{1}} f(x) d x \\
& \geq \theta_{1} \theta_{2} \int_{\max \left(t-a_{2}, t_{1}\right)}^{\min \left(t-a_{1}, t_{2}\right)} d x
\end{aligned}
$$

and the claim follows.

Corollary A.3 If the functions $f$ and $g$ satisfy the assumptions of Lemma A.2 and in addition we see that $a_{1}<a_{1}^{\prime}<a_{2}^{\prime}<a_{2}$, then

$$
\int_{0}^{t} g(a, t) f(t-a) d a \geq \theta_{1} \theta_{2} \min \left(t_{2}-t_{1}, a_{1}^{\prime}-a_{1}, a_{2}-a_{2}^{\prime}\right)=\delta>0
$$

for $t \in\left(a_{1}^{\prime}+t_{1}, a_{2}^{\prime}+t_{2}\right)$

\section{Competing interests}

The authors declare that they have no competing interests.

\section{Authors' contributions}

SR was responsible and worked on the whole text of the paper and presented all proofs in detail. UW participated in discussions concerning presentation and contributed ideas to the biological part of the paper. VK and BOT participated in discussions concerning presentation and contributed ideas to the mathematical part of the paper. All authors read and approved the final manuscript.

\section{Author details}

'Department of Mathematics, Linkoping University, Linkoping, 58381, Sweden. ${ }^{2}$ Department of Physics, Chemistry, and Biology, Linkoping University, Linkoping, 58183, Sweden.

\section{Acknowledgements}

The authors are thankful to the anonymous referees for useful comments and for pointing out interesting literature that tackles similar topics; see $[17,18]$ and [19].

\section{Endnote}

a Problem (1)-(3) with the slightly different assumptions on coefficients is considered in Chipot [1], and existence and uniqueness of the solution is proved. 


\section{References}

1. Chipot, M: On the equations of age-dependent population dynamics. Arch. Ration. Mech. Anal. 82(1), 13-25 (1983)

2. Thomas, CD, et al.: Extinction risk from climate change. Nature 427, 145-148 (2004)

3. Sharpe, FR, Lotka, AJ: A problem in age distribution. Philos. Mag. 21, 435-438 (1911)

4. McKendrick, AG: Application of mathematics to medical problems. Proc. Edinb. Math. Soc. 44, 98-130 (1926)

5. von Foerster, H: Some remarks on changing populations. In: Stohlman, F Jr. (ed.) The Kinetics of Cellular Proliferation, pp. 382-407. Grune \& Stratton, New York (1959)

6. Brauer, F, Castillo-Chavez, C, Castillo-Chavez, C: Mathematical Models in Population Biology and Epidemiology. Springer, New York (2001)

7. Cushing, J: An Introduction to Structured Population Dynamics. SIAM, Philadelphia (1998). doi:10.1137/1.9781611970005

8. Feller, W: On the integral equation of renewal theory. Ann. Math. Stat. 12, 243-267 (1941)

9. Iannelli, M: Mathematical Theory of Age-Structured Population Dynamics. Applied Mathematics Monographs, vol. 7. Giardini editori e stampatori, Pisa (1995)

10. Kot, M: Elements of Mathematical Ecology. Cambridge University Press, Cambridge (2001)

11. Gurtin, ME, MacCamy, RC: Nonlinear age-dependent population dynamics. Arch. Ration. Mech. Anal. 54, 281-300 (1974)

12. Webb, GF: Theory of Nonlinear Age-Dependent Population Dynamics. Dekker, New York (1985)

13. Caswell, H: Matrix Population Models. Wiley, New York (2001)

14. Periodic, CJM: McKendrick equations for age-structured population growth. Comput. Math. Appl. 12(4-5), 513-526 (1986)

15. Tuljapurkar, S: Population dynamics in variable environments. VI. Cyclical environments. Theor. Popul. Biol. 28, 1-17 (1985)

16. Zeidler, E: Nonlinear Functional Analysis and Its Applications I. Springer, New York (1986)

17. Ayadi, MA, Bchatnia, A, Hamouda, M, Messaoudi, S: General decay in a Timoshenko-type system with thermoelasticity with second sound. Adv. Nonlinear Anal. 4(4), 263-284 (2015)

18. Ahmad, S, Le, D: Global and blow up solutions to cross diffusion systems. Adv. Nonlinear Anal. 4(3), $209-219$ (2015)

19. Radulescu, V, Repovs, D: Partial Differential Equations with Variable Exponents: Variational Methods and Qualitative Analysis. Monographs and Research Notes in Mathematics. CRC Press, Boca Raton (2015)

\section{Submit your manuscript to a SpringerOpen ${ }^{\ominus}$ journal and benefit from:}

- Convenient online submission

- Rigorous peer review

- Immediate publication on acceptance

Open access: articles freely available online

- High visibility within the field

- Retaining the copyright to your article 